\title{
Acknowledgement to Reviewers of JCM in 2017
}

\author{
JCM Editorial Office \\ MDPI AG, St. Alban-Anlage 66, 4052 Basel, Switzerland \\ Published: 9 January 2018
}

Peer review is an essential part in the publication process, ensuring that JCM maintains high quality standards for its published papers. In 2017, a total of 116 papers were published in the journal. Thanks to the cooperation of our reviewers, the median time to first decision was 27 days and the median time to publication was 58 days. The editors would like to express their sincere gratitude to the following reviewers for their time and dedication in 2017:

\author{
Abenavoli, Ludovico \\ Aguilera-Aguirre, Leopoldo \\ Akita, Sadanori \\ Albrecht, Jan \\ Alexeyev, Mikhail \\ Amato, Paula \\ Anderson, Amy E. \\ Andrès, Emmanuel \\ Ansa, Benjamin \\ Ansquer, Jean Claude \\ Aransay, Ana M. \\ Ardizzoni, Elisa \\ Arnaout, Bachaar \\ Arriens, Cristina \\ Athyros, Vasilios \\ Bachanova, Veronika \\ Bader, Peter \\ Baghdadi, Ziad D. \\ Bajgoric, Sanjin \\ Bar-Shavit, Zvi \\ Barzilay, Joshua I. \\ Batorova, Angelika \\ Bauer, Natalie \\ Beisswenger, Christoph \\ Benn, Peter \\ Bernabeu, Carmelo \\ Bernardy, Nancy \\ Bernat, James L. \\ Berntorp, Erik \\ Biagi, Carlotta \\ Bilal, Jawad \\ Blatný, Jan \\ Boateng, Joshua S. \\ Boccardo, Francesco \\ Bonfield, Tracey L.
}

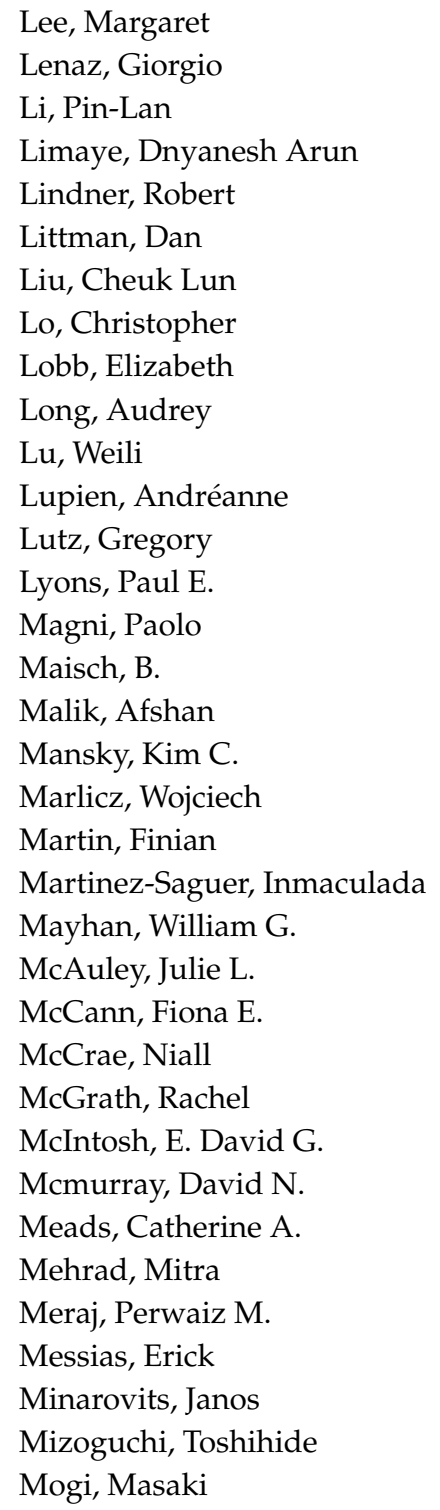


Booth, Stephanie A. Boulware, David R. Bowler, Simon Branchini, Alessio Bratton, Susan L. Bravaccio, Carmela Bricout, Marine Brumeanu, Teodor-Doru Buechler, Christa Bugiardini, Enrico Burger, Charles D. Burtey, Stéphane Calhelha, Ricardo C. Camire, Rodney $\mathrm{M}$.

Carcao, Manuel Chase, Christopher Chen, Yung-Hsiang Chiavaroli, Laura Chiloiro, Giuditta Cho, Mi-La

Christiansen, Christian F.

Ciccone, Marco Matteo

Cicero, Arrigo

Clarke, Kieran

Claydon, Leica Sarah

Cooper, Robert G.

Cortesi, Enrico

Csiszar, Agnes

Daehn, Ilse

Dallenga, Tobias

Dallner, Gustav

Davos, Constantinos

De La Rosa Carrillo, David

De Paepe, Boel

De Paiva, Cintia S.

De Rivera, Joseph

DeBeer, Bryann B.

D'elios, Mario M.

Detweiler, Mark B.

DiMauro, Salvatore

DiMeglio, Linda A.

Dimopoulos, Konstantinos

Doi, Takehiko

Du, Huiyun

Dusi, Veronica

Eiges, Rachel

Eiroa-Orosa, Francisco Jose

Eisenbarth, Hedwig

Eljaafari, Assia

Esteso Díez, Milagros Cristina

Exadaktylos, Aristomenis
Morell, Ferran

Morello, Roy

Morgan, Michael A. M.

Morgan-Warren, Peter J.

Mortiboys, Heather

Mosna, Federico

Moutou, Celine

Narita, Norihiko

Natarajan, Sathish Kumar

Nestler, Ulf

Neureiter, Daniel

Nguyen, T. K. P.

Niyazov, Dmitriy M.

Nogami, Keiji

Nolan, Vikki G.

North, Carol

Obermüller, Nicholas

Page, Anne-Laure

Palaniappan, Balasubramanian

Palla, Roberta

Pantazis, Antonis

Paquis-Flucklinger, Véronique

Parolini, Cinzia

Patel, Indravadan

Pedraza-Chaverri, José

Perarnau, Jean Marc

Pérez-Botero, Juliana

Perricone, Carlo

Peterson, Eliza J. R.

Pfeffer, Gerald

Picca, Stefano

Pointer, Mildred A.

Pokorn, Marko

Pollock, Richard L.

Pozzilli, Paolo

Preissner, Klaus T.

Principe, Maria Ilaria Del

Ramani, Vijay

Randell, Scott H.

Rauch, Bernhard

Raychaudhuri, Siba P.

Ribera, José Maria

Rice, John

Ringström, Gisela

Ro, Eunyoe

Robins, Harlan S.

Rodeghiero, Francesco

Rodenburg, Richard

Rodriguez-Merchan, Emerito Carlos

Roychowdhury, Sanjoy

Rusnati, Marco 
Fadda, Roberta

Favaloro, Emmanuel

Fennell, Kate

Feola, Mauro

Filosto, Massimiliano

Flores-Montero, Juan

Franco, Luis

Fruchtman, Yariv

Gadjeva, Mihaela

Gajula, Rajendra

Garcia-Redondo, Ana Belen

Gebhart, Johanna

Geurts, Jose W.

Giacomin, Paul

Gitelman, Stephen E.

Głąbska, Dominika

Greenberg, Arthur

Gris, Jean Christophe

Hargreaves, Iain

Hatachi, Yukimasa

Heales, Simon

Heitink-Pollé, Katja M. J.

Helmby, Helena

Hendren, Robert

Hohenberger, Gloria

Holme, Pål Andre

Horgan, Graham

Horton, Mark

Horvath, Rita

Hourigan, Christopher

Houston, Mark C.

Ilatovskaya, Daria

Illuminati, Giulio

Im, Sin-Hyeog

Imamura, Teruhiko

Ishibashi, Kenichi

Ishii, Tetsuya

Jeanmart, Stephane

Jensen, Katrin

Jimenez-Rondan, Felix

Jin, Zhongmin

Johnson, Martin K.

Joshi, Pushpa Raj

Jowett, Adam

Kaaja, Risto

Kadlecek, Stephen

Karras, Spiros

Kathrins, Martin
Sakata, Kiyohiko

Sala, Angelo

Salek, Sherveen

Saner, Fuat Hakan

Santulli, Gaetano

Satake, Hironaga

Sawa, Teiji

Schmid, Jean-Paul

Schmitt, Ulrich R.

Seidman, Laura

Sen, Souvik

Senbonmatsu, Takaaki

Shankar, Eswar

Shanmuganathan, Naranie

Sheehan, Vivien

Sherbet, Gajanan V.

Sherwin, LeeAnne B.

Sié, Pierre

Simecka, Jerry W.

Simpson, David A. C.

Singh, Seema

Skapenko, Alla

Smith, Keri

Soiza, Roy L.

Soucie, Mike

Spagnuolo, Rocco

Stanton, Robert C.

Stenmark, Kurt

Stevens, Natalie R.

Sugiyama, Takashi

Sukocheva, Olga

Sukumaran, Anil

Suzuki, Noboru

Svendsen, Morten Bo Søndergaard

Swerdlow, Russell

Szablewski, Leszek

Szolnoky, Győző

Tagarakis, Georgios

Takayama, Yoshiharu

Taube, Joe

Tekle, Michael

Teotia, Pooja

Theilmann, Lorenz

Thoene, Jess

Thoma, Myriam

Thornton, David

Tiano, Luca

Tonolo, Giancarlo 
Kawanami, Daiji

Keipert, Christine

Kneepkens, C. M. Frank

Kofler, Markus

Kojima, Masami

Kok, Victor C.

Kotake, Shigeru

Kotloski, Robert

Kozela, Ewa

Krysinska, Karolina

Kubota, Yoshiaki

Kumar, Gaurav

Kumar, Sajeesh

Kunz, Wolfram

Kurasawa, Kazuhiro

Kurdowska, Anna K.

Kuzmiene, Loreta

Kwakowsky, Andrea

La Rocca, Renato V.

Ladetto, Marco

Ladjemi, Maha Zohra

Lanz, Tobias V.

Larsen, S.

Laubach, Victor E.

Laurent, Louise C.

Lee, Kyung-Yil
Trebicka, Jonel

Tsoulfas, George

Tuck, Caroline

Tulloh, Robert

Van der Velden, Vincent H. J.

Van Dyke, Knox

Van Ryn, Joanne

Vandergheynst, Frédéric

Varetto, Gianfranco

Vork, Lisa

Vyas, Julian

Wada, Jun

Wang, Qiang

Wang, Tsung-Jen

Wang, Yibin

Watson, Paul J.

Weir, Gordon

Williams, Joah L.

Winkler, Johannes

$\mathrm{Wu}$, Wen-Sheng

Wydau, Sandra

Yadollahpour, Ali

Yamakawa, Hideaki

Yin, Jun

Ying, Gui-shuang

Zaheer, Asgar

(C) 2018 by the author. Licensee MDPI, Basel, Switzerland. This article is an open access article distributed under the terms and conditions of the Creative Commons Attribution (CC BY) license (http:/ / creativecommons.org/licenses/by/4.0/). 